\title{
L'analyse par activation de neutrons de réacteur
}

\author{
G. Meyer
}

Laboratoire Pierre Süe, CEA-Saclay, 91191 Gif-sur-Yvette cedex, France

\author{
I- INTRODUCTION \\ I.1- Historique \\ I.2- Principe
}

\section{II- SOURCES DE NEUTRONS- FLUX NEUTRONIQUES}

\section{III- SECTIONS EFFICACES D'ABSORPTION- RESONANCES}

\author{
IV- CALCULS DE CONCENTRATION
}

V- SPECTROMETRIE G ET ANALYSE

\section{VI- EXEMPLES D'APPLICATIONS}

\section{VII- REFERENCES}

\begin{abstract}
Résumé : Quand les neutrons traversent la matière, certains sont transmis sans interaction, les autres interagissent avec le milieu traversé par diffusion et par absorption. Ce phénomène d'absorption est utilisé pour se protéger des neutrons, mais aussi pour les détecter; il peut également être utilisé pour identifier les noyaux "absorbants" et ainsi analyser le milieu traversé. En effet par différentes réactions nucléaires $(\mathrm{n}, \gamma),(\mathrm{n}, \mathrm{p}),(\mathrm{n}, \alpha)$, (n,fission), on obtient des noyaux résiduels qui sont souvent radioactifs; on dit que l'échantillon est "activé". Si l'on connaît le rendement d'activation et donc le pourcentage de noyaux ainsi "transmutés", les mesures de radioactivité induite vont permettre de déterminer la composition de l'échantillon irradié. Cette méthode dite d'analyse par activation neutronique est pratiquée depuis la découverte du neutron. Elle a permis grâce à sa sélectivité et à sa sensibilité d'avoir accès au domaine des traces et des ultra-traces dans des champs d'application très divers comme la métallurgie, l'archéologie, la biologie, la géochimie etc...
\end{abstract}

\section{INTRODUCTION}

\subsection{Historique}

En 1930, Bothe et Becker découvrent l'émission d'un rayonnement très pénétrant provoqué par l'action des $\alpha$ du radium sur des noyaux légers de lithium, de béryllium ou de bore. En 1931, F. Joliot reprend les expériences de Bothe avec une source $\alpha$ de polonium : il observe que le nouveau rayonnement, en traversant une chambre de Wilson remplie d'hydrogène, produit des protons très énergétiques et annonce que le rayonnement de Bothe est constitué de photons $\gamma$ de haute énergie. Un an plus tard, en se basant sur les mêmes expériences que Joliot, James Chadwick, du laboratoire de Rutherford, annonce dans Nature que ce rayonnement est constitué de neutrons, c'est-à-dire des particules de masse égale au proton mais sans charge, ce qui explique qu'il soit si pénétrant. Rutherford avait prévu ce résultat par le calcul dès 1923 . 
En 1936, Hevesy à Copenhague irradie des minerais de terres rares par les neutrons à l'aide d'une source radium-béryllium; après quelques essais infructueux, il obtient une radioactivité qu'il attribue à de nouveaux isotopes radioactifs identifiés par leur période de décroissance : c'est la découverte de l'analyse par activation neutronique.

Dans d'autres laboratoires européens, les expériences d'irradiation aux neutrons se succèdent : Joliot à Paris, Fermi à Rome, Hahn et Meitner à Berlin découvrent presque simultanément la fission de l'uranium, elle même productrice de neutrons. La réaction en chaîne est bientôt maîtrisée, d'abord en laboratoire puis en réacteur. Les premières "piles" expérimentales vont être utilisées comme sources de neutrons avec des flux beaucoup plus intenses que les sources au radium : Zoë (EL1) en 1948 à Fontenay, puis P2 (EL2) en 1952, EL3 et enfin ORPHEE à Saclay font partie de la lignée des réacteurs à eau lourde. Construit également à Saclay, OSIRIS diverge en 1966 et fournit des flux de neutrons encore plus intenses mais moins bien thermalisés car il fonctionne avec de l'eau légère comme modérateur. Construit en 1970 à proximité des réacteurs de Saclay auxquels il est relié par des convoyeurs hydrauliques et pneumatiques, le Laboratoire Pierre Süe devient l'un des mieux équipés au monde. Aujourd'hui encore, de nombreux laboratoires de recherche publics et privés font appel aux neutrons pour analyser des échantillons d'origines très diverses.

\subsection{Principe}

"L'analyse par activation est fondée sur l'identification et la mesure des rayonnements spécifiques de radionucléides formés par réaction nucléaire". Cette définition générale vaut pour toutes les réactions nucléaires obtenues par bombardement de photons, de particules chargées ou de neutrons. Ces derniers comme les photons ont l'avantage de pénétrer dans la matière sans interaction électrostatique et ils vont interagir avec les noyaux de différentes façons : soit ils vont créer des défauts par collision ou diffusion élastique et inélastique, soit ils vont produire des noyaux différents des noyaux cibles par réaction nucléaire. Dans ce dernier cas, il existe encore deux possibilités :

- si le noyau "rédiduel" est stable, tout se passe comme s'il n'y avait pas eu d'activation;

- si le noyau "résiduel" est radioactif, c'est la mesure de son activité qui va permettre de remonter au nombre de noyaux cibles donc d'effectuer un dosage de l'élément considéré.

La technique est d'autant plus efficace que les matériaux usuels s'activent très peu, par exemple le plomb où on va pouvoir mesurer la radioactivité due à l'antimoine ${ }^{122} \mathrm{Sb}$ sans être gêné par la radioactivité quasiment nulle due au plomb [1].Un autre exemple "historique" est le dosage de traces de hafnium dans le zirconium : il est très facile d'activer le hafnium par les neutrons tandis que le zirconium reste pratiquement "transparent" mais la période du radionucléide ${ }^{179 \mathrm{~m}} \mathrm{Hf}$ étant de 19 secondes, il faut prévoir un système de transfert rapide entre le réacteur et le spectromètre, ce qui était réalisé à l'époque avec les moyens du bord. En fait, c'est le mode d'irradiation mais aussi le mode de désintégration et la période de décroissance du nouveau radionucléide qui conditionnent la mesure de radioactivité et donc la faisabilité de l'analyse.

\section{SOURCES DE NEUTRONS - FLUX NEUTRONIQUES}

Les premières sources de neutrons étaient réalisées à partir d'une source $\alpha$ naturelle : radium ou polonium, et d'un élément léger : béryllium, lithium. Les neutrons émis dans ce cas par une réaction nucléaire $(\alpha, n)$ ont une énergie de plusieurs $\mathrm{MeV}$. Plus tard, on fabriquera des sources "isotopiques" grâce à la fission spontanée de nouveaux radioéléments tels que le californium Cf-252. Les flux obtenus sont de l'ordre de $10^{8}$ neutrons. $\mathrm{cm}^{-2} \cdot \mathrm{s}^{-1}$. Ces sources peuvent être utilisées en laboratoire mais leurs applications sont limitées à cause des flux trop faibles.

Les accélérateurs de particules chargées : protons, deutons, peuvent également devenir des sources de neutrons par réactions $(\mathrm{p}, \mathrm{n})$ et $(\mathrm{d}, \mathrm{n})$. La plus connue est la réaction ${ }^{3} H(\mathrm{~d}, \mathrm{n}){ }^{4} \mathrm{He}$ 
utilisée dans des générateurs de neutrons fabriqués industriellement : des deutons de quelques centaines de keV produits par un petit accélérateur suffisent pour cette réaction qui fournit des neutrons de $14 \mathrm{MeV}$. Ces neutrons de haute énergie permettent par exemple de doser l'oxygène par la réaction ${ }^{16} O(n, p){ }^{16} \mathrm{~N}$. L'azote 16 radioactif a une période de 7,3 secondes et il est émetteur $\gamma$ à 6 et $7 \mathrm{MeV}$ : un analyseur équipé d'un générateur de neutrons de $14 \mathrm{Mev}$ capable de distribuer des flux de $10^{8}$ neutrons. $\mathrm{cm}^{-2} \mathrm{~s}^{-1}$ et d'un scintillateur permet de doser l'oxygène avec une bonne sensibilité de l'ordre de quelques milligrammes. Une application connue de ces générateurs "portables" est l'analyse par activation "in situ" dans des puits de forage. Son principal inconvénient est l'utilisation du tritium radioactif comme cible.

Les sources de neutrons les plus intéressantes au niveau de l'intensité des flux sont les réacteurs nucléaires. Certains pays comme le Canada (réacteur SLOWPOKE) et les EtatsUnis (réacteur TRIGA) ont développé des réacteurs de taille modeste réservés à la caractérisation et à l'analyse. En France, on s'est contenté d'utiliser les neutrons délivrés par les réacteurs de recherche du CEA. Dès les premiers mois de "divergence" de Zoë, à partir de 1949 , on a utilisé ses flux de neutrons $\left(10^{10}\right.$ neutrons. $\left.\mathrm{cm}^{-2} \cdot \mathrm{s}^{-1}\right)$ pour tester mais aussi pour analyser des matériaux destinés à la fabrication de nouveaux réacteurs : plomb, acier, graphite, zirconium, dont les critères de pureté allaient être de plus en plus exigeants. Les flux de neutrons de plus en plus intenses ont permis d'atteindre un niveau de sensibilité incomparable par rapport aux techniques usuelles de la chimie analytique, en particulier pour le dosage d'éléments rares : uranium, thorium, lanthanides, tantale, hafnium, or, iridium etc... D'autre part, le Laboratoire Pierre Süe dispose grâce à ses connexions aux réacteurs ORPHEE et OSIRIS de toute la gamme d'énergie des neutrons délivrés par la fission de l'uranium.

Les neutrons de réacteur sont classés suivant leur énergie en trois grands domaines :

- neutrons thermiques agités uniquement par leur température, ils ont une énergie moyenne de $0,025 \mathrm{eV}$ à la température de $300 \mathrm{~K}$;

- neutrons épithermiques entre $1 \mathrm{eV}$ et $0,5 \mathrm{MeV}$; dans ce domaine, apparaissent les niveaux d'excitation qui créent les phénomènes de résonance;

- neutrons rapides d'une énergie supérieure à $0,5 \mathrm{MeV}$; dans un réacteur, ils représentent la fraction des neutrons de fission qui n'ont pas interagi avec le modérateur.

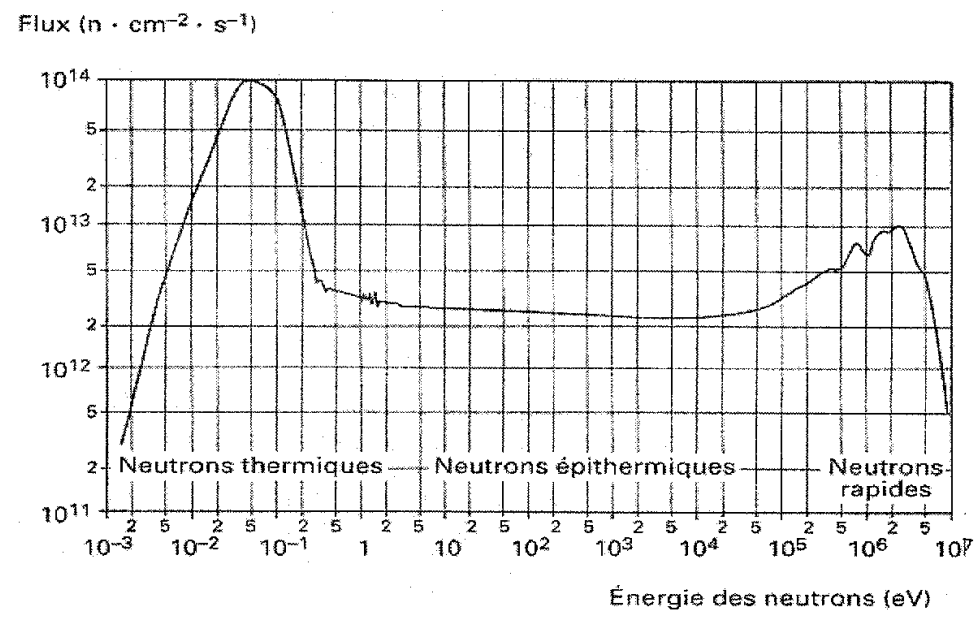

Figure 1 : distribution en énergie des flux de neutrons du réacteur OSIRIS [2].

La figure ci-dessus montre bien l'importance relative des neutrons rapides par rapport au flux thermique pour le réacteur OSIRIS - de 10 à $20 \%$ - : ceci est dû à l'utilisation de l'eau légère 
comme modérateur. En comparaison, le réacteur ORPHEE utilise l'eau lourde qui est un modérateur plus puissant et la composante "rapide" est beaucoup plus faible : de 0,02 à 0,05 $\%$ par rapport au flux thermique.

\section{SECTIONS EFFICACES D'ABSORPTION - RESONANCES}

Quand les neutrons traversent la matière, n'étant porteurs d'aucune charge électrique, ils vont interagir uniquement avec les noyaux de la cible de deux façons différentes : soit ils vont être diffusés en perdant ou non une partie de leur énergie, soit ils vont être absorbés. Il faut donc connaître la probabilité d'interaction : pour cela, on a introduit la notion de section efficace qui est la surface présentée aux particules incidentes par chaque site de réaction. La section efficace totale est la somme des sections efficaces des différents processus d'absorption et de diffusion. Dans notre cas, seule la section efficace d'absorption nous intéresse. La section efficace est la surface utile de la cible vue par les neutrons : elle a donc la dimension d'une surface à l'échelle nucléaire et s'exprime en barns - 1 barn $=10^{-24} \mathrm{~cm}^{-2}$-. A l'intérieur de cette surface, il y a absorption, à l'extérieur de cette surface, la probabilité d'absorption est nulle. On constate que les éléments les plus rares dans la nature ont souvent des sections efficaces d'absorption élevées ( $5.10^{4}$ barns pour Gd) tandis que les éléments "abondants" : $\mathrm{O}, \mathrm{Si}, \mathrm{Fe}$ etc.. ont des sections efficaces faibles $\left(10^{-4}\right.$ barn pour l'oxygène). Cette propriété trouve une explication dans la formation des éléments chimiques par réaction nucléaire : les éléments les plus abondants sont aussi les noyaux les plus stables parce qu'ils ont des sections efficaces d'absorption faibles ou très faibles. Comme le montre le diagramme ci-dessous, le produit de l'abondance $\mathrm{N}$ des éléments sur terre $\left(10^{6}\right.$ pour $\left.\mathrm{Si}\right)$ par la section efficace de capture neutronique $\sigma$ en millibarns est relativement constant :

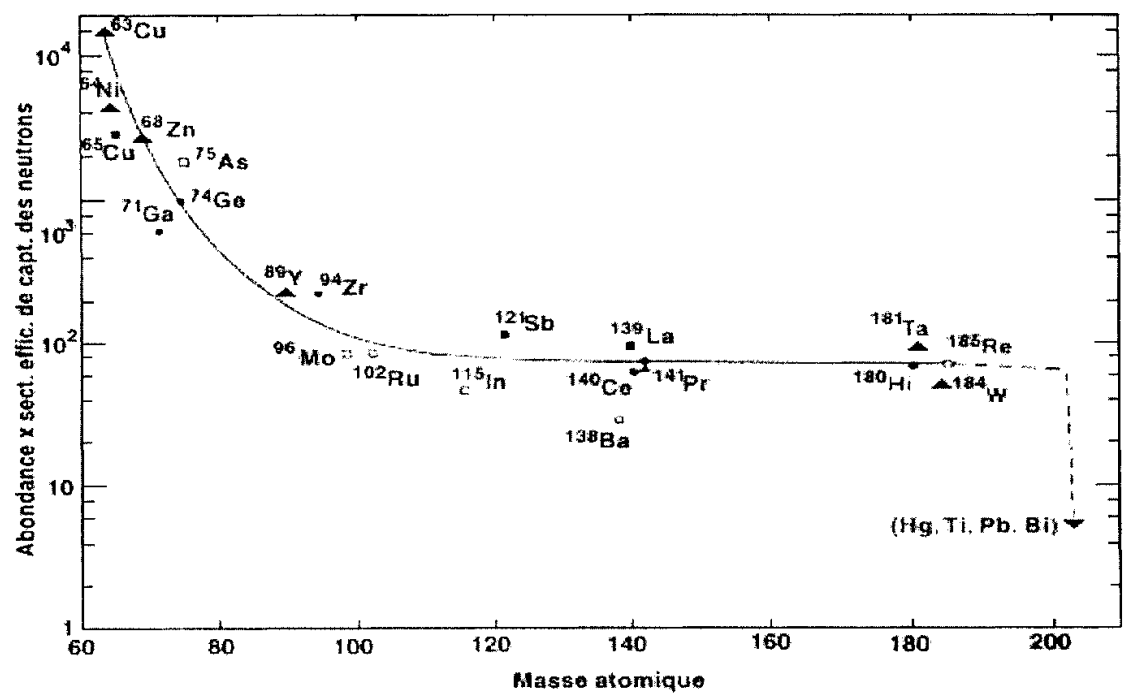

Figure 2 : Variation du produit $\mathrm{N} \sigma$ en fonction de la masse atomique $\mathrm{A}$

L'absorption d'un neutron de faible énergie par un noyau entraîne la formation d'un noyau composé issu de la fusion (noyau + neutron). Après sa formation, le "noyau composé" instable se désintègre en émettant des particules et/ou des photons. Cette théorie a été confortée par de nombreux résultats expérimentaux qui montrent que le mode de désintégration ne dépend que de l'énergie d'excitation du noyau composé c'est-à-dire de 
l'énergie apportée par la particule augmentée de son énergie de liaison. Tout se passe comme si le noyau composé avait perdu la "mémoire" de son mode de formation. L'énergie du neutron incident va donc jouer un rôle important dans la désexcitation du noyau composé et donc la formation du noyau résiduel : si elle est suffisante pour que l'énergie de liaison de certains nucléons soit brisée, l'émission de ces nucléons a lieu. Si l'énergie apportée est faible, la désexcitation s'effectue préférentiellement par émission de photons $\gamma$ : on dit qu'il s'agit d'une capture radiative.

La section efficace d'absorption totale est la section efficace de formation du noyau composé qui va se désintégrer en empruntant plusieurs voies possibles : la section efficace partielle de chaque réaction sera égale à la section efficace de formation du noyau composé pondérée par la probabilité de désintégration de ce noyau en empruntant la voix considérée. Prenons l'exemple de l'absorption des neutrons par l'aluminium qui donne :

$$
{ }^{27} \mathrm{Al}+{ }^{1} \mathrm{n} \longrightarrow{ }^{28} \mathrm{Al}^{*}
$$

Le tableau suivant donne les principales voies possibles de désintégration du noyau composé ${ }^{28} \mathrm{Al}^{*}$ :

Tableau : réactions nucléaires des neutrons sur Al-27

\begin{tabular}{llllll}
\hline Réaction & $\sigma$ (barns) & $\mathbf{Q}(\mathrm{MeV})$ & Produit & Type d'émission & Période \\
\hline$\left(n, n^{\prime}\right)$ & 0,8 & & Al-27 excité & & \\
$(n, \gamma)$ & 0,23 & $+7,7$ (pas de seuil) & Al-28 & $\beta-, \gamma$ & $i 2,23$ minutes \\
$(n, p)$ & 0,03 & $-1,9$ (seuil) & $\mathrm{Mg}-27$ & $\beta-, \gamma$ & 9,45 minutes \\
$(n, \alpha)$ & 0,001 & $-3,14$ (seuil) & $\mathrm{Na}-24$ & $\beta-, \gamma$ & 15,02 heures \\
$(n, 2 n)$ & 0,0 & -13 (seuil) & Al-26 & $\beta+, \gamma, \mathrm{CE}$ & 730000 ans \\
\hline
\end{tabular}

Au niveau énergétique, on définit $Q$ comme étant la différence d'énergie entre le neutron et le noyau cible d'un côté, la particule émise et le noyau résiduel de l'autre. On note que seule la réaction $(\mathrm{n}, \gamma)$ a un bilan en énergie $\mathrm{Q}$ positif c'est-à-dire qu'elle sera possible avec un apport d'énergie faible. Dans ce cas, il n'y a pas de rupture de liaison à l'intérieur du noyau composé et le noyau résiduel Al-28 est identique au noyau composé. La section effícace de 0,23 barns est donnée pour les neutrons thermiques mais elle décroît fortement avec l'énergie des neutrons : à $5 \mathrm{MeV}$, elle n'est plus que de $5 \cdot 10^{-5}$ barn. Les autres désintégrations du noyau composé sont en compétition avec la capture radiative : elles nécessitent un apport d'énergie plus important pour que les particules émises puissent rompre leurs liaisons nucléaires et franchir la barrière coulombienne. Les sections efficaces des réactions à seuil sont données pour des neutrons de $5 \mathrm{MeV}$. La somme des sections efficaces correspondant à toutes les voies de désintégration du noyau composé donne la section efficace d'absorption.

Pour l'absorption des neutrons comme pour les autres réactions nucléaires, on observe des phénomènes de résonance, c'est-à-dire des grandes valeurs de section efficace pour des valeurs précises d'énergie incidente. Par exemple, pour l'absorption des neutrons par une cible d'iridium, on observe une résonance : pour une énergie des neutrons de $0,65 \mathrm{eV}$ on a une section efficace de 4500 barns contre 900 barns à $0,5 \mathrm{eV}$. L'existence de ces phénomènes de résonance a pu être expliquée par les niveaux excités du noyau composé. La largeur à mihauteur de la résonance pour la réaction ${ }^{191} \operatorname{Ir}(\mathrm{n}, \gamma){ }^{192} \mathrm{Ir}$ est la largeur du niveau du noyau composé responsable de cette grande section efficace : elle est d'environ $0,1 \mathrm{eV}$, ce qui correspond à une durée de vie de $10^{-15}$ seconde, beaucoup plus longue que la durée d'un choc simple entre le neutron et le noyau de ${ }^{191}$ Ir qui serait de l'ordre de $10^{-22}$ seconde. Ces 
phénomènes de résonance sont utilisés lors des irradiation derrière un filtre en cadmium dans un réacteur tel qu'Osiris où le flux épithermique est élevé. Le cadmium par sa forte section efficace de capture en neutrons thermiques permet de les absorber sélectivement et de favoriser les réactions résonantes obtenues par les neutrons épithermiques.

\section{CALCULS DE CONCENTRATION}

Pour un noyau de section efficace $\sigma$ - exprimé généralement en barns : 1 barn $=10^{-24} \mathrm{~cm}^{-2}$ bombardé par des neutrons de flux $\Phi$ ( en neutrons. $\mathrm{cm}^{-2} . \mathrm{s}^{-1}$ ), le taux d'absorption sera égal au produit $\sigma . \Phi$. Pour $\mathrm{N}_{0}$ noyaux irradiés pendant un temps dt, le nombre de noyaux radioactifs créés est $\mathrm{N}_{0} . \sigma$. $\Phi$.dt et en tenant compte de la décroissance radioactive de probabilité $\lambda$ on obtient une variation :

$$
\mathrm{dN}=\mathrm{N}_{0} \sigma \Phi \mathrm{dt}-\lambda \mathrm{Ndt}
$$

L'équation différentielle : $\quad \frac{d N}{d t}+\lambda \mathrm{N}=\mathrm{N}_{0} \sigma \Phi$

admet une solution simple:

$$
\begin{aligned}
\mathrm{N} & =\frac{N_{0}}{\lambda} \sigma \Phi\left(1-e^{-\lambda t}\right) \\
\frac{d N}{d t} & =\mathrm{N}_{0} \sigma \Phi\left(1-e^{-\lambda t}\right)
\end{aligned}
$$

qui s'écrit aussi:

Si le temps d'irradiation $t$ est faible devant la période de décroissance radioactive $T=\frac{\ln 2}{\lambda}$, ce qui est très souvent le cas, on peut simplifier en :

$$
\frac{d N}{d t}=\mathrm{N}_{0} \sigma \Phi \frac{t \ln 2}{T}
$$

La mesure de la radioactivité en fin d'irradiation $\frac{d N}{d t}$ et la connaissance des autres paramètres permettent de calculer $\mathrm{N}_{0}$ donc d'effectuer un dosage. Prenons l'exemple de l'iridium : pour une radioactivité mesurée de $1000 \mathrm{~Bq}$ (désintégrations par seconde) sur l'isotope le plus favorable ${ }^{192}$ Ir dans un échantillon irradié 24 heures dans le réacteur ORPHEE, on calcule une masse d'iridium d'environ 10 nanogrammes. Ceci donne une idée de la sensibilité de l'analyse par activation neutronique pour le dosage de cet élément. La sensibilité de l'analyse par activation neutronique pour un élément donné sera d'abord déterminée par le produit de la section efficace de capture par l'abondance relative de l'isotope le plus favorable. En effet cette section efficace peut être très différente suivant les isotopes du même élément. Ainsi pour le cadmium qui possède huit isotopes stables, l'un d'entre eux ${ }^{113} \mathrm{Cd}$ possède une section élevée de 20600 barns pour une abondance de $12,3 \%$ tandis que les autres ont une section efficace beaucoup plus faible: 0,3 barn pour le plus abondant $(28,8 \%){ }^{114} \mathrm{Cd}$. Pour évaluer le pouvoir absorbant du cadmium, on calculera la section efficace globale qui est la somme des sections des différents isotopes pondérées par leur abondance relative soit 2530 barns.

\section{SPECTROMETRIE $\gamma$ ET ANALYSE}

La plupart des radionucléides obtenus par activation vont se désexciter par émission $\beta$ due à l'excès de neutrons suivie d'une ou plusieurs émissions $\gamma$. Prenons l'exemple du dosage du cobalt dans un acier. Par capture radiative $(n, \gamma)$, le seul isotope stable du cobalt ${ }^{59} \mathrm{Co}$ va donner deux isotopes : ${ }^{60 \mathrm{~m}} \mathrm{Co}$ de 10,5 minutes de période et son isomère ${ }^{60} \mathrm{Co}$ de période beaucoup plus longue : 5,27 ans. La section efficace de production des deux isotopes est 
sensiblement la même : 19 barns. On peut donc envisager de doser le cobalt par spectrométrie de ${ }^{60 \mathrm{~m}} \mathrm{Co}$ dont la période est favorable.

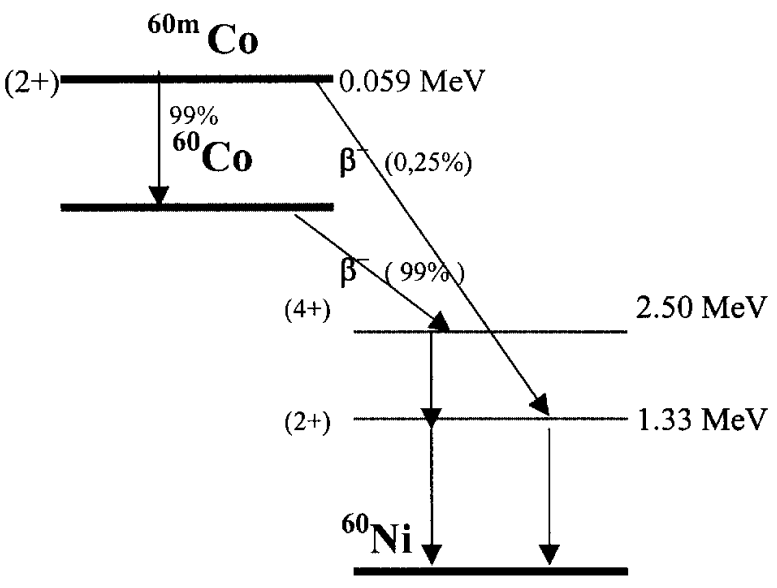

Figure 3 : schéma de désintégration du cobalt :

${ }^{60 \mathrm{~m}} \mathrm{Co}$ : période 10,47 minutes -Transition isomérique $99 \%$ - désintégration $\beta^{-} 0,25 \%$

${ }^{60} \mathrm{Co}$ : période 5,26 ans - émission $\beta^{-} 99,9 \% \operatorname{Emax} 318 \mathrm{keV}$.

En fait, la transition isomérique de $59 \mathrm{keV}$ entre ${ }^{60 \mathrm{~m}} \mathrm{Co}$ et ${ }^{60} \mathrm{Co}(99 \%$ d'embranchement $)$ est presque entièrement convertie en $\mathrm{X}$ et électrons de conversion $(98 \%)$. La mesure de radioactivité de ${ }^{60 \mathrm{~m}} \mathrm{Co}$ par spectrométrie $\gamma$ est donc impossible et il faudra utiliser son descendant ${ }^{60} \mathrm{Co}$ qui émet deux photons $\gamma$ très intenses (100\%) d'énergie 1,17 et $1,33 \mathrm{MeV}$. Dans un tel dosage, il faut aussi tenir compte de la radioactivité induite dans la matrice, ici par activation du fer. Le seul isotope gênant est ${ }^{59} \mathrm{Fe}$ qui émet à 1,1 et à $1,3 \mathrm{MeV}$ mais la section efficace de capture de 1,1 barn et surtout l'abondance isotopique faible de $0,28 \%$ de ${ }^{58} \mathrm{Fe}$ permettent d'obtenir une radioactivité due à la matrice relativement faible. Enfin, la résolution en énergie des détecteurs semi-conducteurs $\left(2 \mathrm{keV}\right.$ sur la raie à $\left.1332 \mathrm{keV} \mathrm{de}{ }^{60} \mathrm{Co}\right)$ permet de séparer complètement les raies à 1,17 et $1,33 \mathrm{Mev}$ de ${ }^{60} \mathrm{Co}$ de celles à 1,1 et 1,29 $\mathrm{Mev}$ provenant de la décroissance de ${ }^{59} \mathrm{Fe}$. On peut donc envisager un dosage instrumental du cobalt dans l'acier avec une très bonne sensibilité de l'ordre du microgramme.

$\mathrm{Si}$ on utilise pour la détection un scintillateur $\mathrm{NaI}$, la résolution en énergie n'est plus suffisante pour séparer les différentes raies. Deux techniques sont alors envisageables:

- l'une radiochimique qui consiste à séparer chimiquement le cobalt du fer; une séparation chromatographique permet cette opération [3];

- l'autre instrumentale qui consiste à mesurer simultanément sur deux scintillateurs les impulsions provenant des deux émissions en cascade à 1,17 et $1,33 \mathrm{MeV}$ profitant du fait que les émissions à 1,1 et $1,3 \mathrm{Mev}$ de ${ }^{59} \mathrm{Fe}$ sont non-coïncidantes ou indépendantes dans le temps. C'est la technique dite de "comptage en coïncidence" 
Pour améliorer la sensibilité donc l'erreur statistique sur un pic d'absorption $\gamma$, il faudra réduire le fond continu du spectre dû à la diffusion des photons dans le détecteur. Pour cela, on peut utiliser une technique instrumentale de comptage en anti-coïncidence ou une séparation chimique après l'irradiation dans la mesure ou la durée de vie du noyau résiduel le permet.

L'analyse par activation est ainsi devenue une technique instrumentale qui permet de doser une vingtaine d'éléments traces dans n'importe quel échantillon solide avec un seuil de détection compris entre le nanogramme et le microgramme (voir spectre ci-dessous).

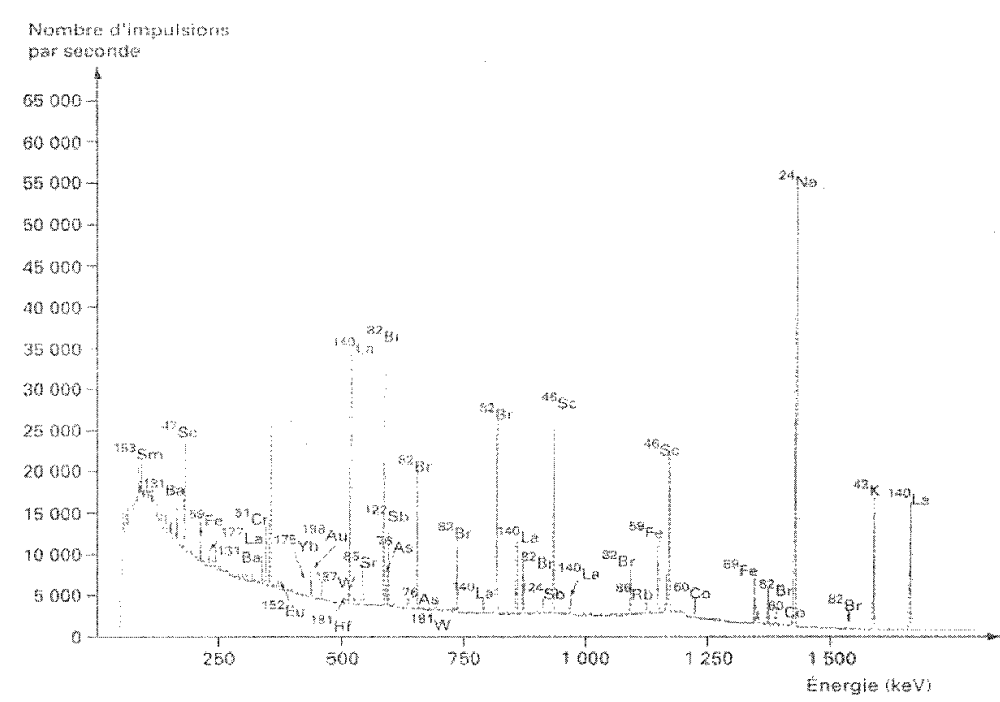

Figure 4 : spectre gamma d'un échantillon de lichen irradié aux neutrons [2]

La technique de spectrométrie de coïncidence a été mise à profit pour le dosage ultra-sensible de l'iridium tout d'abord dans les météorites puis dans les matériaux terrestres où les teneurs sont généralement inférieures à la ppb $\left(\mathrm{mg}_{\mathrm{kg}}{ }^{-1}\right)$. Nous avons également testé avec succès la méthode pour le dosage de l'étain, du sélénium etc.. [4] On observe sur le spectre biparamétrique ci-dessous, les coïncidences principales de ${ }^{192} \mathrm{Ir}$ entre les émissions $\gamma$ de 316 et $468 \mathrm{keV}$. Le spectre est construit par comptage simultané sur deux voies de mesure et un enregistrement des évènements "coïncidents" à l'intérieur d'une certaine fenêtre de temps. On voit que par cette technique, on supprime les évènements simples. Par contre on ne supprime pas les phénomènes de diffusion qui créent aussi des coïncidences. 


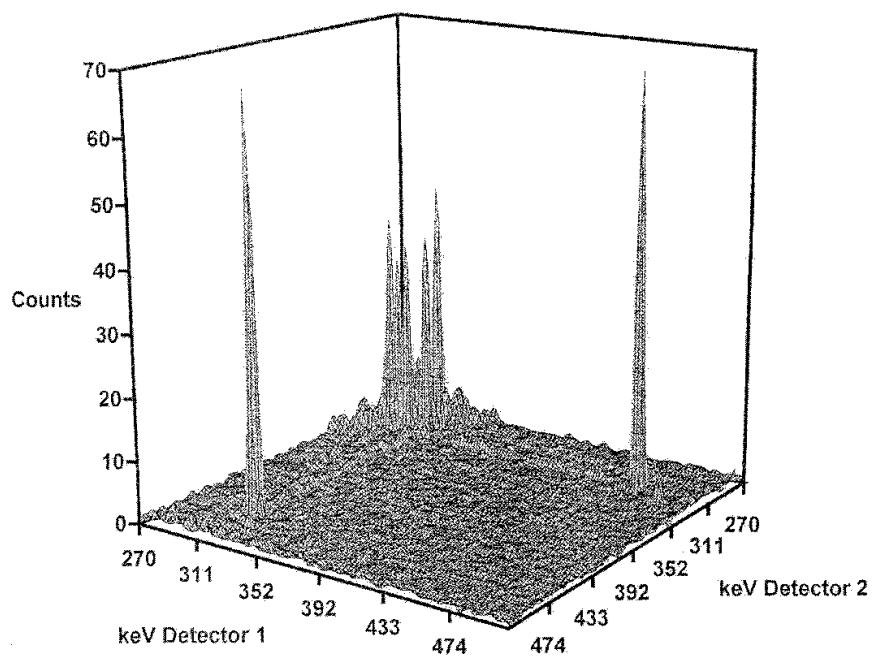

Figure 5 : spectre biparamétrique de ${ }^{192}$ Ir dans une roche irradiée aux neutrons [5]

\section{EXEMPLES D'APPLICATION}

Dès la découverte du neutron et de la radioactivité artificielle, l'analyse par activation neutronique va devenir la méthode de choix pour l'identification et le dosage des éléments en trace dans des matériaux ultra-purs tels que les métaux, les semi-conducteurs, les films plastiques et les matériaux spécifiques du nucléaire : graphite, plomb, zirconium etc... Dans le laboratoire de Joliot, au Collège de France, un chimiste dénommé Pierre Süe développe ces nouvelles méthodes d'analyse par activation aux neutrons mais aussi aux protons, deutons et même aux photons. C'est Joliot lui-même qui suggérera d'utiliser la technique pour doser l'arsenic dans l'instruction du célèbre procès de Marie Besnard. Plus récemment, on a à nouveau fait appel à l'analyse par activation neutronique pour tenter de démontrer que Napoléon avait été empoisonné à l'arsenic. Mais ces résultats très "médiatisés" ne doivent pas occulter la somme considérable de données sur les éléments traces dans toutes sortes de matériaux archéologiques, géologiques et biologiques. Le Laboratoire Pierre Süe spécialisé en activation neutronique a largement participé à l'acquisition de ces données.

A partir des années 70, une toute nouvelle science baptisée géochimie va utiliser la sensibilité de l'activation neutronique pour s'ouvrir de nouvelles possibilités d'investigation dans le domaine des traces [6]. L'utilisation des sections efficaces de résonance trouve ici un champ d'application très intéressant en permettant de mieux doser et donc de mieux comprendre le 
comportement des éléments en faible ou très faible concentration dans la genèse des roches. Les installations du Laboratoire Pierre Süe ont permis l'utilisation intensive de la technique d'irradiation derrière filtre en cadmium très profitable grâce aux flux épithermiques élevés du réacteur OSIRIS, ouvrant ainsi le champ d'investigation des géochimistes à de nouveaux éléments tels que : $\mathrm{U}, \mathrm{Th}, \mathrm{Ta}, \mathrm{Hf}$ etc... [7]. L'étude complémentaire des concentrations en éléments traces et des rapports isotopiques va faire avancer de façon considérable la connaissance des différents réservoirs de la géosphère et de leurs interactions. Par exemple, les terres rares ou lanthanides vont être utilisés comme marqueurs de ces réservoirs. La normalisation à la composition des chondrites qui sont des météorites censées représenter le matériau originel du système terrestre va permettre de mettre en évidence la différenciation des différents domaines constitutifs de notre planète actuelle : manteau, croûte continentale, plancher océanique...

L'analyse par activation neutronique est aussi à l'origine de la découverte de la fameuse anomalie en iridium des sédiments de la limite Crétacé-Tertiaire qui va permettre à L. Alvarez de faire une publication retentissante [8]. Dans cette publication, l'anomalie en iridium - de quelques nanogrammes par gramme - va être interprétée comme étant la signature géochimique d'un cataclysme d'origine extra-terrestre. Ceci est basé sur la constatation de l'absence d'iridium dans les sédiments terrestres et de son abondance dans la matière cosmique. Mais c'est la sensibilité extrême du dosage de cet élément par activation neutronique qui a permis à L. Alvarez de déceler la présence d'iridium et d'avancer sa théorie très contestée dans un premier temps mais aujourd'hui quasiment admise par tous les spécialistes.

\section{Références}

1. P. Süe, Bull. Soc. Chim., 18, 9 (1951).

2. G. Revel - Extrait de la Collection "Techniques de l'Ingénieur"- Traité "Analyse et caractérisation" - P 2565 (1999).

3. P. Albert, M. Caron, G. Chaudron, C.R. Acad. Sci. Paris, 236,1030 (1953)

4. G. Meyer, J. Radioanal. Nucl. Chem. 114, 223 (1987)

5. H. Huber, C. Koeberl, I. McDonald, W.U. Reimold, J. Radioanal. Nucl. Chem., 244, 603 (2000).

6. C.J. Allègre, M. Treuil, J.F. Minster, B. Minster et F. Albarede, Contr. Mineral. Petrol. , 60, 57-75 (1977).

7. B. Chayla, H. Jaffrezic et J.-L. Joron, C. R. Acad. Sc. Paris, t. 277, Série D, 273-275 (1973).

8. L.W. Alvarez, W. Alvarez, F. Asaro, H.V. Michel, Science, 208, 1095 (1980). 